\title{
Características reproductivas de las mujeres consultantes en servicios de urgencia general de la VIII Región
}

\author{
Iván Q uevedo $\mathrm{L}^{1}$, Maritza Flores $\mathrm{O}^{2}$, Marcelo Castillo $\mathrm{N}^{3}$. \\ Reproductive history of women \\ consulting in emergency services \\ in a southern region of Chile
}

Background: The occurrence and timing of menstrual and reproductive events such as menarche, regularity of menses, births, maternal breastfeeding and menopause play an important role in a woman's life. Aim: To study the variability of reproductive live from menarche to menopause in different age groups in Chilean Caucasian and Mapuche aborigine females in a southern location of Chile. Subjects and Methods: A questionnaire about reproductive history was answered by 502 women consulting in six general emergency rooms of different public hospitals of the $8^{\text {th }}$ region of Chile, between May and September 2002. Results: The median age at menarche was 12.8 years among adolescents and 13.7 years among older women ( $\mathrm{p}<0.001$ ). Fecundity rate was 5.2, 3.9 and 1.8 among older women, adults and young adults, respectively. Forty seven percent of adolescent had at least one child. The median age for natural menopause was 47 years. Eighty one percent of older women and $65 \%$ of adult women had given breastfeeding for more than six months. No statistical differences in reproductive characteristics were observed between Chilean Caucasians and Mapuche aborigines. Conclusions: Among these women, the age of menarche in younger women is similar to that of women from industrialized countries. Fecundity has been progressively decreasing. Menopause tends to occur sooner than in other geographical regions, probably due to the high number of surgical sterilizations in our population. Adolescent pregnancy still is a problem (Rev Méd Chile 2005; 133: 929-34).

(Key Words: Menarche; Menopause; Reproductive behavior; Reproduction)

Recibido el 14 de octubre, 2004. Aceptado el 4 de abril, 2005.

${ }^{1}$ Departamentos de Medicina Interna, ${ }^{2}$ Salud Pública y de ${ }^{3}$ Especialidades Médicas, Facultad de Medicina, Universidad de Concepción.

L os eventos que se desarrollan a lo largo de la vida reproductiva de la mujer tales como la menarquia, la regularidad del ciclo menstrual, el

Correspondencia a: Dr. Iván Quevedo L Avda. Andalué $\mathrm{N}^{\circ}$ 1825, San Pedro de la Paz, Concepción Chile. E mail: equevedo@udec.cl número de hijos, duración de la lactancia y la menopausia se han relacionado con algunas patologías de gran impacto en la Salud Pública como son la cardiopatía coronaria ${ }^{1}$ y el cáncer de mama ${ }^{2-4}$.

Por otro lado, durante el potencial período reproductivo la mayoría de las mujeres requiere del apoyo de los servicios sociales y de salud, 
debido a la necesidad de una adecuada planificación familiar, prevención de las enfermedades de transmisión sexual, además de la atención del parto, del pre y post natal ${ }^{5}$.

Todos los eventos anteriormente mencionados tienen, por lo tanto, enormes implicancias socioeconómicas para el país, y los cambios de estas características hacen necesario, en muchas ocasiones, replantear el enfoque de las políticas educativas, como por ejemplo el abordaje de las relaciones sexuales en la adolescencia, prevención del embarazo en este grupo etáreo $0^{6,7} 0$ la necesidad de una atención de salud focalizada a la mujer climatérica ${ }^{8}$.

La edad reproductiva, que abarca el período que va desde la menarquia a la menopausia, varía considerablemente entre los diferentes países y grupos étnicos ${ }^{9}$.

Estudios previos han mostrado que la menarquia se presenta más tempranamente en los países desarrollados, lo que se ha relacionado con la mejoría en las condiciones de vida de esos países durante el siglo pasado ${ }^{11}$. Existen datos controversiales en relación a si la menopausia se modifica según país o etnia, postulando algunos autores que estaría inversamente relacionada con la fertilidad y directamente con la esperanza de vida ${ }^{11}$.

La población de la VIII región de Chile constituye el 12,3\% de la población del país. Sociocultural y étnicamente, la población de la ciudad de Concepción, donde se ubica la capital regional, no difiere del resto del país, sin embargo en la Provincia de Arauco existe un alto porcentaje de población de origen mapuche. Esta distribución demográfica permite analizar comparativamente el comportamiento de las características reproductivas en las mujeres de ambos grupos ${ }^{12}$.

El objetivo de este estudio fue describir las caracteństicas de la vida reproductiva de la mujer consultante en los servicios de urgencia general en la VIII región (menarquia, regularidad del ciclo menstrual, paridad, duración de la lactancia, anticoncepción, menopausia) y determinar si estos eventos podrían estar influenciados por factores culturales como la etnia, hábito tabáquico o la escolaridad.

\section{Pacientes y MÉtodos}

El estudio se realizó en la VIII región de Chile entre los meses de mayo y septiembre de 2002.
Se encuestó, al azar, a 513 pacientes mujeres chilenas entre 15 y 90 años, que concurrieron espontáneamente a consultar a los servicios de urgencia general de los hospitales Guillermo Grant Benavente de Concepción, Las Higueras de Talcahuano y Florida, en la Provincia de Concepción y Hospitales de Curanilahue, Cañete y Contulmo, en la Provincia de Arauco. A todas las pacientes se les solicitó su consentimiento informado. Con el fin de obtener una información objetiva, se aplicó un test de detección de trastorno cognitivo (Minimental Test) ${ }^{13,14}$ a todas las pacientes mayores de 65 años. Se excluyó a todas las que tuvieron un puntaje menor a 21 . Del grupo encuestado, 11 mujeres presentaron un Minimental Test alterado, por lo que fueron excluidas del estudio, quedando conformada la muestra por 502 mujeres.

El cuestionario aplicado incluyó datos relacionados con la vida reproductiva de la mujer como: edad de la menarquia, regularidad del ciclo menstrual, paridad, duración de la lactancia, uso y tipo de método de planificación familiar comúnmente utilizados, como los anticonceptivos orales (ACO) o los dispositivos intrauterinos (DIU), edad de la menopausia y si ésta fue quirúrgica 0 espontánea. Además, se registró los años de escolaridad (básica, media o universitaria), hábito tabáquico y etnia (mapuche o chilena).

Se separó la población según grandes grupo de edad, quedando conformado de la siguiente manera: adolescentes (menores de 21 años), adultas jóvenes (entre 21 y 45 años), adultas (entre 46 y 65 años) y adultas mayores (mayores de 65 años) ${ }^{15}$.

Análisis estadístico y definición de variables. Para el análisis estadístico se utilizó el programa SPSS $11.0^{16}$. Las variables continuas fueron presentadas a través de promedios y desviación estándar.

Edad de la menarquia se consideró a la edad de comienzo de las menstruaciones. Se definió como ciclo menstrual regular a aquellos entre 26 y 36 días entre el comienzo de un ciclo y otro, que además no tuvieran diferencias de más de tres días en el patrón basal de sus ciclos. Paridad se definió como el número de hijos nacidos vivos. La duración de la lactancia se refirió a si ésta era prolongada o no, la lactancia prolongada se consideró como una lactancia mayor a seis meses. Los métodos de planificación 
familiar se definieron como uso de anticonceptivos orales o dispositivos intrauterinos. Las mujeres que presentaban menopausia, se definieron como aquellas que referían un cese espontáneo de las menstruaciones, por un período mayor a 12 meses antes de la aplicación de la encuesta. La variable escolaridad se basó en que si había cursado educación básica, media o superior (universitaria o instituto de educación superior). El hábito tabáquico fue definido como un consumo mayor a tres cigarrillos/ día por un período mínimo de diez años, o que estuviese fumando uno o más cigarrillos diarios al momento de la aplicación de la encuesta. Perteneciente a la etnia mapuche se consideró aquellas mujeres con dos apellidos de origen mapuche, con presencia de rasgos característicos y que se considerara perteneciente a esa cultura (Oyarce A. Definition of Ethnic Culture: Contribution to Epidemiological Research. Annals of the IV. Latinclen Meeting. Santo Domingo, Septiembre 1997).

\section{RESUlTADOS}

Los resultados del cuestionario aplicado a las 513 mujeres fueron analizados. La edad promedio de las 502 pacientes seleccionadas fue de 48,8 años (rango 15 a 90 años). Treinta y nueve pacientes (7,8\%) eran adolescentes, 189 (37,7\%) eran adultas jóvenes, 154 (30,7\%) correspondía al grupo adulto y las adultas mayores fueron 120 (23,9\%). La distribución según grupo étnico mostró que 27 pacientes, es decir, 5,4\% pertenecía a la etnia mapuche. La edad promedio de la menarquia fue de 13,8 $\pm 1,6$ años. En la Figura 1 se muestra la edad de la menarquia por grupo etáreo, siendo en el grupo de menores de 25 años de 12,8 \pm 1 años y en las adultas mayores de $13,7 \pm 1,6$ años, encontrándose una diferencia estadísticamente significativa entre ambos grupos ( $p<0,001)$. No se encontró diferencias significativas según etnia. Sesenta y siete pacientes $(13,3 \%)$ refirieron tener ciclos menstruales irregulares, cifra que aumentaba a 17,9\% en el grupo de adolescentes. El hecho de esta mayor prevalencia de irregularidades del ciclo menstrual en las adolescentes, podría explicarse porque el eje gonadal aún no está completamente maduro durante este período.

En relación a la población de mujeres que habían presentado su menopausia, ésta correspondió a 250 (50,4\%); de éstas, 39 (15,4\%) tuvieron una menopausia quirúrgica. Entre las mujeres que tuvieron una menopausia espontánea, la edad promedio fue $47 \pm 5$ años, edad significativamente

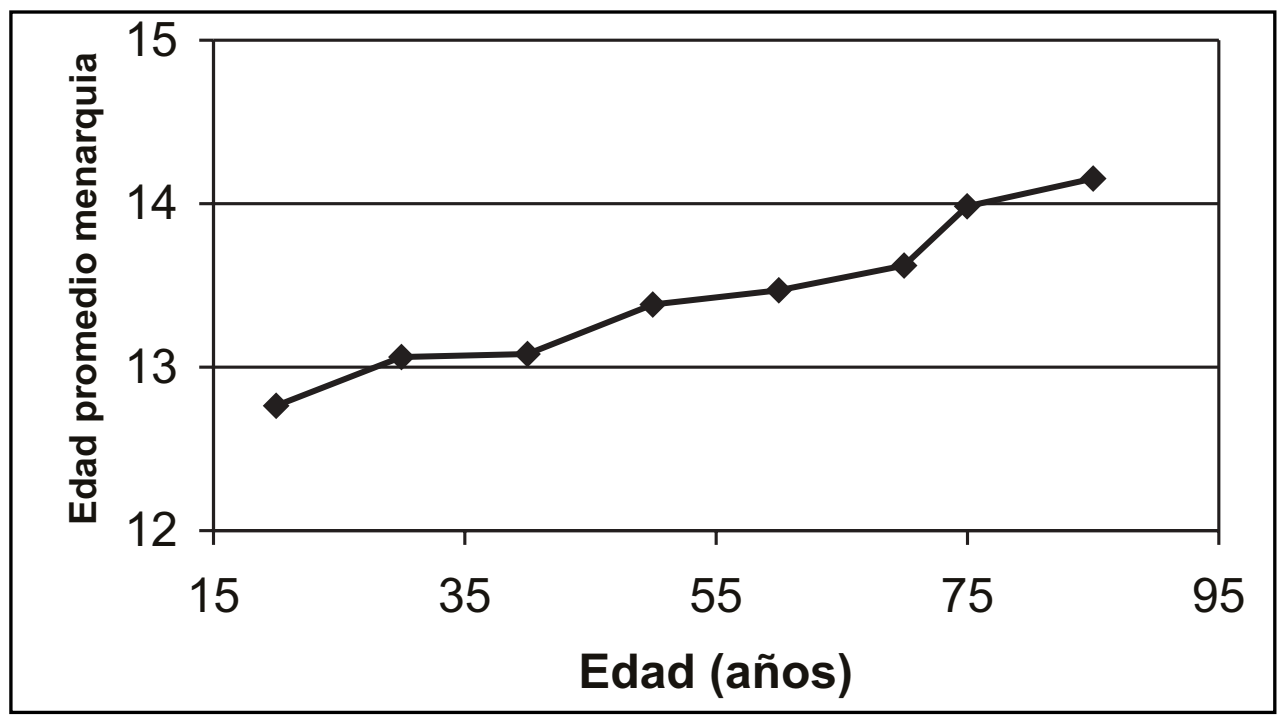

Figura 1. Edad promedio de la menarquia por grupo etáreo. 
Tabla 1. N úmero promedio de hijos por grupo etáreo

\begin{tabular}{|lcccrr|}
\hline $\begin{array}{l}\text { Edad } \\
\text { en grupos) }\end{array}$ & Promedio & $\begin{array}{c}\text { Desviación } \\
\text { estándar }\end{array}$ & Mínimo & Máximo & N \\
\hline Adolescentes & 0,54 & 0,555 & 0 & 2 & 39 \\
Adulto joven & 1,87 & 1,375 & 0 & 7 & 189 \\
Adulto & 3,89 & 2,408 & 0 & 13 & 154 \\
Adulto mayor & 5,23 & 3,471 & 0 & 15 & 120 \\
Total & 3,19 & 2,779 & 0 & 15 & 502 \\
\hline
\end{tabular}

mayor a las que tuvieron una menopausia quirúrgica $40,3 \pm 6,9$ años ( $p<0,001)$. No se encontró diferencias significativas en la edad de la menopausia al estudiar los diferentes grupos según escolaridad, menarquia, número de hijos, duración de la lactancia, método anticonceptivo o hábito tabáquico. Sin embargo, en la etnia mapuche la edad de la menopausia se presentó a más temprana edad

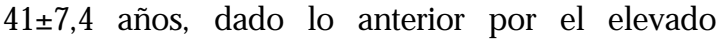
porcentaje $(53,3 \%)$ de mujeres mapuches que tenían una menopausia quirúrgica.

Las usuarias de método de planificación familiar fueron 379 (75,5\%). En el grupo de adolescentes, $35,1 \%$ refirió tener alguno de los tipos de método de planificación familiar consultados en el cuestionario. Llama la atención que sólo 8 (6,6\%) mujeres adultas mayores manifestaron haber utilizado durante su vida fértil algún método de planificación familiar, esto se debe probablemente a que no existían políticas de planificación familiar durante la juventud de estas mujeres. Los métodos de planificación familiar analizados se distribuyeron en una forma homogénea, los ACO y los DIU fueron utilizados por $47,8 \%$ y $52,2 \%$ de las mujeres, respectivamente. En el cuestionario no se consideró la abstinencia periódica, los métodos de barrera o inyectables. La esterilización tubaria fue utilizada por 69 mujeres y de éstas 60 eran mayores de 40 años.

En la Tabla 1 se ve la distribución de la población según el número de hijos por grupo etáreo, observándose un aumento progresivo en el número de hijos por mujer conforme aumenta la edad.

$\mathrm{Al}$ analizar la distribución de la lactancia (Tabla 2), se observó que de las 418 mujeres que habían dado de lactar, 64,8\% lo había realizado por más
Tabla 2. M ujeres encuestadas según duración lactancia materna

\begin{tabular}{|lrr|}
\hline $\begin{array}{l}\text { Duración lactancia } \\
\text { materna }\end{array}$ & $\mathrm{N}^{\circ}$ & $\%$ \\
\hline Mayor a 6 meses & 271 & 64,8 \\
Menor a 6 meses & 147 & 35,2 \\
Total & 418 & 100,0 \\
\hline
\end{tabular}

de 6 meses. Sin embargo, en el grupo de las adultas mayores, este número se incrementó significativamente ya que $81,5 \%$ de ellas dio de lactar por más de 6 meses ( $p<0,001)$.

\section{DisCUSIÓN}

Los resultados de nuestro estudio muestran que diversos aspectos en las características reproductivas de las mujeres de la VIII región, se modifican significativamente al analizar los diferentes grupos etáreos.

La menarquia se ha adelantado, presentándose actualmente en nuestras adolescentes a edades similares a la de países desarrollados como Estados Unidos $^{17}$ y Suiza ${ }^{5}$. Similar observación, en relación al adelanto del desarrollo puberal en las niñas, ha sido realizado en nuestro país por el Grupo del Instituto de Investigaciones Materno Infantil de la Universidad de Chile $^{18}$. Si bien no están claras las causas del adelanto de la menarquia, se ha sugerido que este fenómeno se debería a una constante mejońa de las condiciones de vida de la población 
durante las últimas décadas, asociado a un aumento en la prevalencia de la obesidad. Esto también ha sido observado en los países industrializados durante la segunda mitad del siglo pasado ${ }^{19,20}$. La edad promedio de la menopausia espontánea en nuestra población fue $47 \pm 5$ años, no variando significativamente según grupo etáreo. La edad promedio de la menopausia en nuestra población fue inferior a los 50 años descrita en la literatura ${ }^{9}$, incluso fue inferior a países con características étnicas y socioculturales similares al nuestro como Colombia y Méjico cuyas edades promedios de la menopausia son 50 y 51 años, respectivamente ${ }^{21,22}$. La edad de la menopausia en nuestra población es cercana a la de 49 años que tiene la República Popular China. El hecho que ambas poblaciones presenten menopausias a edades más tempranas que el resto de la población mundial, podría ser explicado por el elevado número de mujeres de nuestra población que han sido sometidas a una esterilización tubaria $(13,7 \%)$, tal como sucede con las políticas de control de la natalidad en la República Popular China ${ }^{23}$.

En nuestro trabajo, del universo de mujeres consultantes en los servicios de urgencia general, 47,4\% tenía más de 50 años. Esta alta prevalencia de mujeres mayores de 50 años, creemos refleja el envejecimiento que está experimentando nuestra población en las últimas décadas. Esta observación lleva a plantear que debería tomarse en cuenta la problemática en salud de un grupo etáreo en permanente crecimiento ${ }^{24}$.

La fertilidad en las mujeres consultantes en los servicios de urgencia evidencia una constante y marcada disminución, al igual que la tendencia general de la Región de las Aménicas ${ }^{25}$. El número de hijos observado en nuestra población adulta es de 3,9, significativamente menor en comparación con los 5,2 hijos de nuestra población adulta mayor. Aun si se considera que el grupo de las adultas jóvenes tiene vida fértil en proceso, pero dado que el número promedio de hijos de este grupo es de sólo 1,8, nos hace proyectar que en el futuro permanecerá la tendencia descendente de la fecundidad. Por otro lado, nuestro estudio mostró que casi la mitad (47\%) de las adolescentes consultantes espontáneas en los servicios de urgencia tienen al menos un hijo. En la encuesta no se consultó si era una mujer sexualmente activa. Sin embargo, esta elevada proporción de madres adolescentes podnía ser explicado porque sólo un tercio de éstas $(35,1 \%)$ utilizaban algún método de planificación familiar, y que nuestra muestra fue obtenida de adolescentes consultantes en hospitales públicos que normalmente pertenecen a una población con bajo nivel socioeconómico ${ }^{26}$. Este fenómeno de una disminución general de la fecundidad, con persistencia de una alta prevalencia de embarazos en adolescentes, es un problema observado en la mayonía de los países de las Américas e incluso en países industrializados como Estados Unidos, en poblaciones de origen afroamericano e hispánico que tienen un escaso desarrollo sociocultural $^{27}$. Similar observación ha sido realizada en Chile, en una investigación en ocho comunas de la Región Metropolitana, que mostró que el embarazo en la adolescente, en proporción, es cinco veces mayor en estratos socioeconómicos bajos que en los sectores socioeconómicos altos ${ }^{28}$.

Una elevada proporción de las mujeres consultantes en los servicios de urgencia $(64,8 \%)$ han dado de lactar por al menos seis meses. Nuestra encuesta no consideró si la lactancia materna había sido exclusiva, predominante o complementada. La proporción de mujeres de nuestra población que ha dado de lactar, es inferior a 85\% de prevalencia de lactancia materna observado en algunos consultorios urbanos de la atención primaria en la Región Metropolitana. Sin embargo, nuestra población logra llegar al objetivo planteado por nuestro país en 1992, de llegar a fines de esa década con $80 \%$ de lactancia materna exclusiva a los 4 meses y de $35 \%$ de lactancia complementada al año de vida ${ }^{29}$. El hecho que en el grupo de las adultas mayores, el 81,5\% refirió haber dado de lactar por más de seis meses, hace suponer que existen cambios socioculturales como el ingreso de la mujer al campo laboral, que imparten negativamente en lograr una lactancia exclusiva hasta los seis meses.

En conclusión, nuestro trabajo muestra que existen diferencias importantes en las características reproductivas de las mujeres consultantes en los servicios de urgencia de la VIII región al analizar y comparar los diferentes grupos etáreos. La menarquia se presenta en nuestras adolescentes a edades similares a la de los países industrializados, la fecundidad muestra una persistente tendencia a la baja y existe una población creciente de mujeres mayores consultando en los servicios de urgencia. Aún persisten problemas característicos de país en vía de desarrollo, como el embarazo en la adolescente. 


\section{REFERENCIAS}

1. Ness RB, Schotiand HM, Flegal KM, Sjofer FS. Reproductive history and coronary heart disease risk in women. Epidemiol Rev 1994; 16: 298-314.

2. Harris JR, Lippman ME, Veronesi U, WiLet W. Breast cancer. N Engl J Med 1992; 327: 319-28.

3. KeiseY JL, Gammon MD, JoHn EM. Reproductive factors and breast cancer. Epidemiol Rev 1993; 15: 36-47.

4. KeLSEY JL, HorN-Ross PL Breast cancer: Magnitude of the problem and descriptive epidemiology. Epidemiol Rev 1993; 15: 7-16.

5. Morabia A, Khatchatrian N, Bernstein M, WalKer DM, Campana A. Reproductive characteristics of a population of urban Swiss women. Acta Obstet Gynecol Scand 1996; 75: 838-42.

6. Molna R, Sandoval J, Luengo X. Adolescencia y Embarazo. En: Pérez-Sánchez A, Donoso F. Obstetricia. 3a Ed. Santiago, Chile: Ed. Mediteráneo, 1999.

7. Marfán J. Adolescencia: Desarrollo Afectivo y Sexual. En: Pérez Sánchez. Ginecología. 3a Ed. Santiago, Chile: Ed. Mediterráneo, 2003.

8. WikLUND I. How to evaluate Quality of Life» in menopause? En: Birkhauser MH. European Consensus devolopment conference on menopause. Monteux: Eska Ed., 1994.

9. Morabia A, Costanza MC. International variability in ages at menarche, first livebirth and menopause. Am J Epidemiol 1998; 148: 1195-205.

10. HaRLow SD, Ephross SA. Epidemiology of menstruation and its relevance to women's health. Epidemiol Rev 1995; 17: 265-86.

11. World Health Organization Scientific Group. Technical report series. Geneva, Switzerland: World Health Organization 1996; 866: 14-6.

12. Censo de Población y Vivienda. Instituto Nacional de Estadística. Chile, 2002.

13. Prince M, Acosta D, Chiyu H, Scazufica M, Varghese M, for the 10/66 Dementia Research Group. Dementia diagnosis in developing countries: a cross cultural validation study. The Lancet 2003; 361: 909-17.

14. Quiroga P, Aibala C, Kiafsen G. Validación de un test de tamizaje para el diagnóstico de demencia asociada a edad, en Chile. Rev Méd Chile 2004; 132: 467-78.

15. Papalia DE, Olds SW. Psychology New York; MacGraw-Hill, 1998.

16. SPSS Inc., 223 South Wacker Drive, $11^{\text {th }}$ Floor, Chicago, Il. 60606-6307, EE.UU.

17. Bean JA, Leeper JD, Wallace RB, Sherman BM, JAGGER H. Variations in the reporting of menstrual histories. Am J Epidemiol 1979; 109: 181-5.

18. Gaete $X$, Unanue M, Avila A, Cassoria F. Cambios en la edad de inicio de la pubertad en niños de la comuna de Santiago: Implicancias para el diagnóstico de la pubertad precoz. Rev Chil Pediatr 2002; 73: 363-72.

19. Morabia A, Bernstein M, Herttier S, Yш A. A community-based surveillance of cardiovascular risk factors en Geneva: methods, resulting distributions, and comparisons with other populations. Prev Med 1997; 26: 311-9.

20. Kaplowitz P, SLora E. Earlier onset of puberty in girls: Relation to increased body mass index and race. Pediatrics 2001; 108: 347-53.

21. Cure-Cure C, Cure-Ramírez P, Teran E, LópezJARAMLLO P. Bone-mass peak in multiparity and reduced risk of bone-fractures in menopause. Int J Gynaecol Obstet 2002; 45: 247-57.

22. Sievert LL, Hautaniemi SL. Age at menopause in Puebla, Mexico. Human Biol 2003; 75: 205-26.

23. Peng P. The population of China: Problems and strategy. Chine Popul Today 1992; 9: 1-10.

24. PARRA M. Realidad demográfica en Latinoamérica y tratamiento hormonal de reemplazo. En Menopausia y longevidad. Eds. O González C, E Arteaga U, P. Contreras C. Ediciones Sociedad Chilena de Climatenio 1999; 74-85.

25. OPS/OMS. Programa Análisis de Situación de Salud, División de Salud y Desarrollo, Condiciones de Salud, Condiciones de Salud en las Américas, indicadores básicos 997, Washington, DC. 1997.

26. FNUAP. Fondo de Naciones Unidades para actividades de población. Foro abierto de Salud y Derechos Reproductivos. Diagnóstico de salud reproductiva en Chile. Primera Edición, 1997. Editores Váldes T, Faúndez A.

27. Celade: América Latina: Tasa de fecundidad por edad, 1950-2025. Boletín demográfico 1993; 26 (52).

28. Chadwick M, Hamel P, Vergara B. Proyecto embarazo en Adolescentes. Sernam-Unicef. Santiago de Chile, 1992.

29. Ilabaca J, Atalah E. Comparación de la prevalencia y porcentaje de acuerdo entre dos métodos de análisis de la lactancia matema. Rev Med Pediatr 2002; 73: 583-9.

\section{Agradecimientos}

Los autores agradecen la valiosa colaboración en la redacción y ordenamiento del material manuscrito de la Sra. Ana Cristina Contreras, Secretaria Centro Saval de Concepción. 